\title{
Perfil antropométrico de pré-escolares de uma creche em Duque de Caxias, Rio de Janeiro
}

Anthropometric profile of preschool children of a day-care center in Duque de Caxias, Rio de Janeiro, Brazil

\author{
Ana Lilian B. dos Santos ${ }^{1}$, Leila Sicupira C. S. Leão
}

\section{RESUMO}

Objetivo: Descrever o perfil antropométrico de préescolares matriculados em uma creche de Duque de Caxias, Rio de Janeiro.

Métodos: Estudo transversal descritivo, com aferição de medidas antropométricas (peso e estatura) e preenchimento de um questionário socioeconômico. A população estudada foi composta por 33 pré-escolares menores de seis anos de idade matriculados na creche. A população de referência para comparação do grupo estudado foi a do National Center for Health Statistic (NCHS) e da Organização Mundial de Saúde (OMS).

Resultados: Observou-se que a maioria (48,5\%) das crianças fazia parte da classe econômica C. Nenhuma delas apresentou deficit pôndero-estatural ( $Z$ escore $<-2)$ de acordo com os índices estatura/idade (E/I), peso/idade (P/I) e peso/ estatura (P/E). Entretanto, 21,2\% apresentaram risco de baixa estatura ( $Z$ escore entre -2 e -1); 15,2 e 9,1\% mostraram risco de baixo peso, segundo os índices $\mathrm{P} / \mathrm{I}$ e $\mathrm{P} / \mathrm{E}$, respectivamente; $21,2 \%$ para sobrepeso ( $Z$ escore entre $+1 \mathrm{e}+2$ ) tanto para o índice $\mathrm{P} / \mathrm{I}$ quanto para o índice $\mathrm{P} / \mathrm{E}$ e, finalmente, 3,0 e $6,1 \%$ apresentaram obesidade $(Z$ escore $>+2$ ) segundo os índices $\mathrm{P} / \mathrm{I}$ e $\mathrm{P} / \mathrm{E}$, respectivamente.

Conclusões: Reflexos da transição nutricional foram observados neste estudo, no qual se encontrou ausência de deficits pôndero-estaturais indicativos de desnutrição e um considerável excesso de peso, indicativo de sobrepeso e obesidade.

Palavras-chave: antropometria; estado nutricional; estatura; peso corporal; pré-escolar.

\section{ABSTRACT}

Objective: To describe the anthropometric profile of preschool children registered in a day-care center in Duque de Caxias, Rio de Janeiro, Brazil.

Methods: This cross sectional study enrolled 33 preschool children less than six years old registered in a day care center. All families were asked to answer a socioeconomic questionnaire and the children were assessed regarding weight and height. The National Center for Health Statistic (NCHS) and World Health Organization (WHO) growth charts were considered as standards for comparison purposes.

Results: The majority of children belonged to medium socioeconomic group (C class). None of them presented height-for-age or weight-for-age deficits with $\mathrm{Z}$ score $<-2$. However, $21.2 \%$ presented risk for low height (-2 to $-1 \mathrm{Z}$ score); 15.2 and $9.1 \%$ presented risk of low weight according to weight-for-age and weight-for-height respectively; $21.2 \%$ of the children had overweight ( +1 to $+2 \mathrm{Z}$ scores) according to both indexes, and finally, 3.0 and $6.1 \%$ presented obesity ( $>+2 \mathrm{Z}$ scores) according to weight-for-age and weight-forheight respectively.

Conclusions: Nutritional transition seems to be present in the studied children, with absence of indicators of malnutrition and a considerable frequency of overweight and obesity.

Key-words: anthropometry; nutritional status; body height; body weight; child preschool.
'Graduada em Nutrição pela Universidade Federal do Estado do Rio de Janeiro (UNIRio), Rio de Janeiro, RJ, Brasil

2Professora assistente da Escola de Nutrição da UNIRio, Rio de Janeiro, RJ, Brasil
Endereço para correspondência:

Ana Lilian B. dos Santos

Avenida Parque Águas Claras, quadra 301, conj. 08, lote 05/07, apto. 1004 - Norte

CEP 71902-180 - Águas Claras/DF

E-mail: analilian_nutricao@yahoo.com.br

Recebido em: 28/11/2007

Aprovado em: 25/5/2008 


\section{Introdução}

O estado nutricional de uma criança possui papel fundamental para que seu crescimento seja progressivo e para que ela desenvolva suas aptidões psicomotoras e sociais. Alterações de deficit ou excesso expõem tais crianças a riscos potenciais de agravos à saúde, bem como a futuros problemas de relações interpessoais e funcionais dentro da comunidade.

Os resultados dos três últimos inquéritos nacionais sobre o estado nutricional de crianças brasileiras apontam para um declínio da prevalência de desnutrição em crianças menores de cinco anos de idade ${ }^{(1-3)}$. Entretanto, esta tendência encontra-se em um contexto epidemiológico chamado de transição nutricional, no qual há a substituição dos deficits pelos excessos nutricionais ${ }^{(4,5)}$.

No meio urbano, a grande dificuldade das mães em compatibilizar o emprego com o cuidado infantil impulsionou a criação de espaços destinados ao atendimento das crianças (creches), que constituem um importante recurso para viabilizar sua participação no mercado de trabalho. A importância das creches no perfil de transição nutricional mencionado anteriormente está no fato de as crianças permanecerem na creche oito a dez horas por dia e, durante este tempo, receberem dois terços de suas necessidades nutricionais ${ }^{(6)}$.

Desta forma, o presente trabalho tem como objetivo geral descrever o perfil antropométrico dos pré-escolares de uma creche localizada na cidade de Duque de Caxias, Rio de Janeiro. Os objetivos específicos do estudo são: determinar a freqüência das alterações nutricionais de acordo com os índices estatura/idade (E/I), peso/idade (P/I) e peso/estatura $(\mathrm{P} / \mathrm{E})$; analisar comparativamente a curva de crescimento pôndero-estatural das crianças da creche com as curvas do National Center for Health Statistic (NCHS) ${ }^{(7)}$ e da Organização Mundial de Saúde (OMS) ${ }^{(8)}$; comparar a prevalência de deficit de peso encontrada na creche com os resultados da Pesquisa de Orçamentos Familiares ${ }^{(3)}$ e relacionar o estado nutricional com a classificação econômica da família, baseada em dados socioeconômicos (poder aquisitivo da família e grau de escolaridade dos responsáveis).

\section{Métodos}

Foi realizado um estudo de delineamento transversal descritivo para determinar o perfil antropométrico dos préescolares matriculados em uma creche localizada no bairro de Gramacho, em Duque de Caxias, Rio de Janeiro.
O critério adotado para a inclusão dos participantes foi a autorização do responsável, por meio da assinatura de um termo de consentimento, o preenchimento do questionário sobre informações socioeconômicas e a presença do préescolar nos dias da coleta de dados. O pré-escolar que não atendeu a um desses critérios foi excluído do estudo. O trabalho foi aprovado pelo diretor da creche onde o estudo foi realizado, por meio da assinatura de uma declaração de autorização da realização da pesquisa na creche.

Dentre as variáveis investigadas, foram consideradas de interesse neste estudo: estado socioeconômico, sexo, idade, peso e estatura. A fim de determinar o poder aquisitivo das famílias e o grau de escolaridade dos responsáveis, foi utilizado o critério de classificação econômica proposto pela Associação Brasileira de Empresas de Pesquisa (ABEP) em $2003^{(9)}$. A partir deste instrumento, construiu-se um questionário sobre posse de bens e grau de instrução do chefe de família, que foi entregue ao responsável para ser preenchido e assinado.

O registro correspondente ao sexo dos pré-escolares foi definida pela observação da presença de características próprias de cada gênero, enquanto que a idade das crianças em meses foi definido pela busca do dia, mês e ano de nascimento nas fichas disponíveis na secretaria da creche.

Para a coleta da variável peso, o equipamento antropométrico consistiu de balança portátil digital da marca Plenna ${ }^{\circledR}$, com capacidade de $150 \mathrm{~kg}$ e precisão de $100 \mathrm{~g}$. Essa balança foi submetida a um teste de calibração baseado no método de comparação de pesos-padrão com as indicações do instrumento calibrado, conforme procedimento para balança eletrônica, tendo sido aprovada de acordo com a Portaria 236/94 do Inmetro $^{(10)}$. A balança foi colocada sobre superfície plana no vestiário da creche, sendo o peso obtido por medição única. As crianças foram pesadas apenas com roupa íntima e sem calçados. Para isso, permaneciam de pé no centro da balança, com o peso corporal igualmente distribuído entre ambos os pés ${ }^{(11)}$.

Para aferir a estatura por medição única, foi utilizado o estadiômetro de madeira com precisão de $0,1 \mathrm{~cm}$, fixado verticalmente em uma parede lisa e sem rodapé. As crianças foram posicionadas com calcanhares, glúteos e dorso da cabeça contra o estadiômetro e braços para baixo e relaxados. A cabeça foi posicionada de tal modo que o plano de Frankfort ficava paralelo ao solo ${ }^{(11)}$. O plano anatômico de Frankfort estende-se desde a margem inferior da órbita ocular até a margem superior do meato auditivo ${ }^{(12)}$.

A partir das informações sobre a estatura, o peso, a idade e o sexo, determinaram-se os valores de E/I, P/I e P/E. O 
estado nutricional foi avaliado a partir do cálculo do escore Z para os índices E/I, P/I e P/E, de acordo com as populações de referência do $\mathrm{NCHS}^{(7)}$ e $\mathrm{OMS}^{(8)}$, visto que tais curvas também foram referências nos estudos utilizados na discussão deste trabalho.

Para a classificação do estado nutricional segundo os índices $\mathrm{P} / \mathrm{I}$ e $\mathrm{P} / \mathrm{E}$, adotaram-se os seguintes pontos de corte: escore $Z<-2=$ baixo peso; escore $Z$ entre -2 e $-1=$ risco de baixo peso; escore $Z$ entre -1 e $+1=$ eutrofia; escore $Z$ entre +1 e $+2=$ sobrepeso e escore $Z>+2=$ obesidade. Já para a classificação baseada no índice $\mathrm{E} / \mathrm{I}$, os pontos de corte foram: escore $\mathbf{Z}<-2$ = baixa estatura; escore $Z$ entre -2 e -1 = risco de baixa estatura e escore $Z>-1=$ eutrofia. Quando o indicador E/I é alto, não possui significado importante para a saúde pública. Esse indicador normalmente está atrelado a fatores genéticos $^{(13)}$. Os casos individualizados podem apresentar algum interesse em termos clínicos, mas não em termos de saúde pública. Em função disso, o indicador E/I alto não foi valorizado nos resultados e na discussão.

Para a análise estatística dos resultados, as variáveis foram inseridas no programa estatístico Excel 2003 e analisadas

Tabela 1 - Classificação do estado nutricional dos pré-escolares da creche segundo o índice estatura/idade (E/I)

\begin{tabular}{lrr}
\hline \multirow{2}{*}{ Estado nutricional } & \multicolumn{2}{c}{ E/I } \\
\cline { 2 - 3 } & $\mathbf{n}$ & $\%$ \\
\hline Baixa estatura & 0 & 0 \\
Risco de baixa estatura & 7 & 28 \\
Eutrófico & 18 & 72 \\
\hline Total & 25 & 100 \\
\hline
\end{tabular}

Tabela 2 - Classificação do estado nutricional dos pré-escolares da creche segundo os índices peso/idade (P/I) e peso/estatura $(\mathrm{P} / \mathrm{E})$

\begin{tabular}{lcccc}
\hline \multirow{2}{*}{ Estado nutricional } & \multicolumn{2}{c}{ P/I } & \multicolumn{2}{c}{ P/E } \\
\cline { 2 - 5 } & $\mathbf{n}$ & $\%$ & $\mathbf{n}$ & $\%$ \\
\hline Baixo peso & 0 & 0 & 0 & 0 \\
Risco de baixo peso & 5 & 15,2 & 3 & 9,1 \\
Eutrófico & 20 & 60,6 & 21 & 63,6 \\
Sobrepeso & 7 & 21,2 & 7 & 21,2 \\
Obesidade & 1 & 3 & 2 & 6,1 \\
\hline Total & 33 & 100 & 33 & 100 \\
\hline
\end{tabular}

no programa Statistical Package for the Social Sciences (SPSS) 12.0. Os resultados foram apresentados como medidas de tendência central e dispersão. As diferenças entre os gêneros foram comparadas por meio do teste $t d e$ Student.

\section{Resultados}

Estavam matriculadas na creche 65 crianças no dia da coleta dos dados relativos a sexo e data de nascimento. Desse total, 32 crianças foram excluídas por terem se desvinculado da creche até o dia da coleta das medidas de peso e estatura (seis crianças) ou pelo não preenchimento do questionário de classificação econômica ou assinatura do termo de consentimento pelos responsáveis ( 26 crianças). Portanto, a população estudada constou de 33 crianças, sendo 16 do sexo feminino e 17 do masculino. Deste universo, 14 (42\%) tinham faixa etária de 33 a 46 meses, 13 (39\%) entre 46,1 a 59 meses e seis (18\%) entre 59,1 a 72 meses. A distribuição, segundo o peso, apresentou 17 crianças (52\%) na faixa de peso de 11,6 a $16,8 \mathrm{~kg}, 12(36 \%)$ entre $17,4 \mathrm{a} 21,4 \mathrm{~kg}$ e quatro (12\%) entre 22 a $26,2 \mathrm{~kg}$. Segundo a estatura, a distribuição apresentou 13 crianças (39\%) entre 90,5 a 102cm, 17 (52\%) com 102,2 a $112 \mathrm{~cm}$ e três entre 112,8 a $128,8 \mathrm{~cm}$. Tanto a idade quanto o peso e a estatura não apresentaram diferenças significantes $(p<0,05)$ entre meninos e meninas, quando as variáveis foram comparadas pelo teste $t$.

Dessa forma, a análise dos resultados para os índices E/I, $\mathrm{P} / \mathrm{I}$ e $\mathrm{P} / \mathrm{E}$ foram aplicadas para a amostra total. Em relação ao índice E/I obtido para cada criança da creche e comparado aos dados antropométricos encontrados na população de referência do $\mathrm{NCHS}^{(7)}$, observou-se uma prevalência de risco de baixa estatura de 28\% (Tabela 1). Já para o índice P/I, a prevalência de sobrepeso (21\%) foi maior do que a de risco de baixo peso (15\%), com freqüência de obesidade de 3\% (Tabela 2). Em relação ao índice P/E, constatou-se que, do total de crianças, $9 \%$ apresentaram risco de baixo peso, $21 \%$ estavam com sobrepeso e $6 \%$ eram obesas (Tabela 2).

Utilizando os resultados de estatura, peso e idade, foram construídas três curvas (E/I, P/I e P/E) para meninas e meninos da creche. Essas curvas foram comparadas com as curvas construídas a partir das medianas de estatura e peso do $\mathrm{NCHS}^{(7)}$ e da $\mathrm{OMS}^{(8)}$ (Gráficos 1 a 6). As curvas dos índices E/I, P/I e P/E geradas a partir das medianas de referência do $\mathrm{NCHS}^{(7)}$ e da $\mathrm{OMS}^{(8)}$ apresentaram comportamentos semelhantes, tanto para meninas quanto para meninos. $\mathrm{Na}$ comparação dos resultados encontrados no grupo estudado e nas populações de referência ${ }^{(7,8)}$, pode-se observar a proxi- 


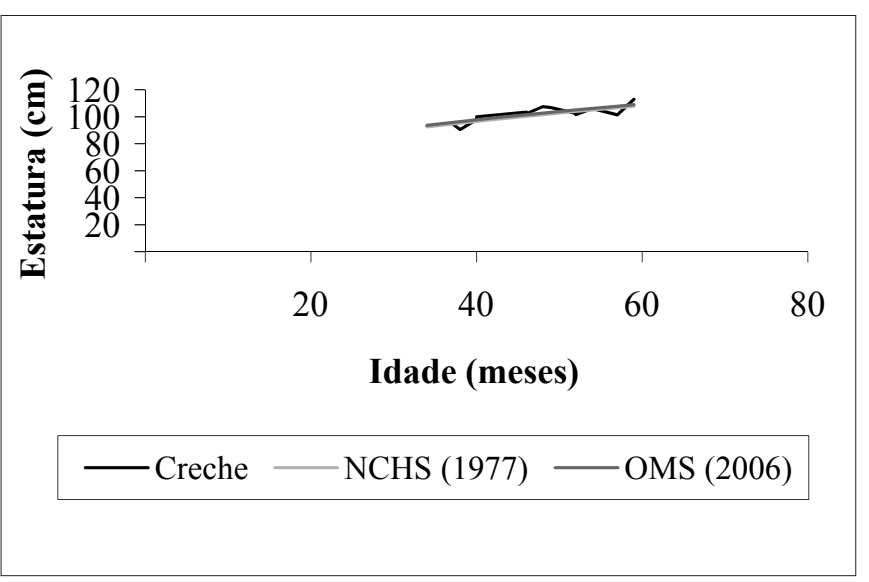

Gráfico 1 - Comparação da curva de estatura/idade observada nas meninas da creche com as curvas NCHS e OMS

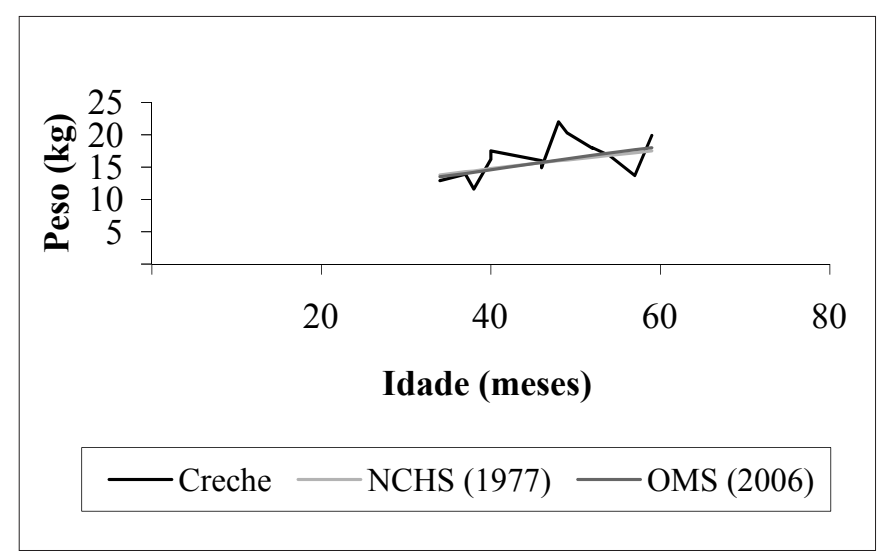

Gráfico 3 - Comparação da curva de peso para idade observada nas meninas da creche com as curvas NCHS e OMS

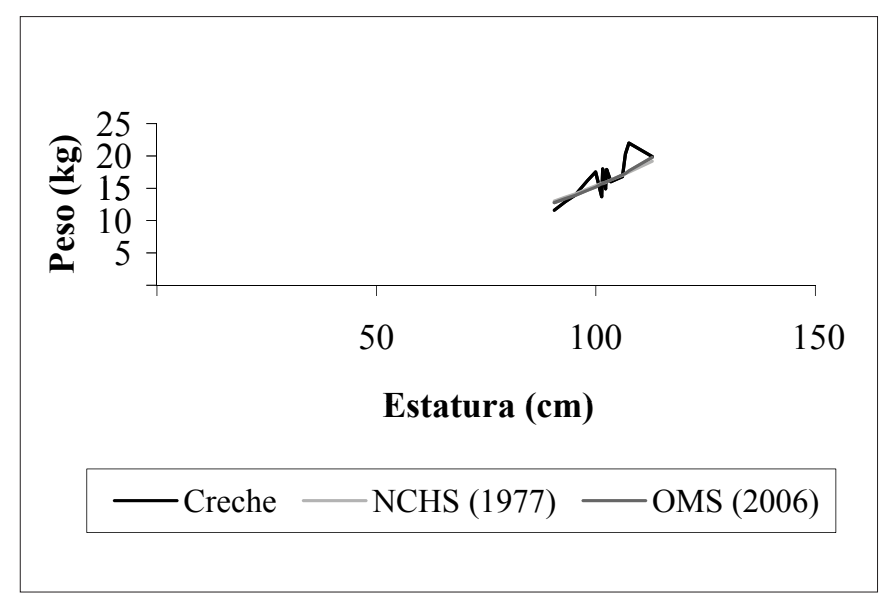

Gráfico 5- Comparação da curva de peso e estatura observada nas meninas da creche com as curvas NCHS e OMS

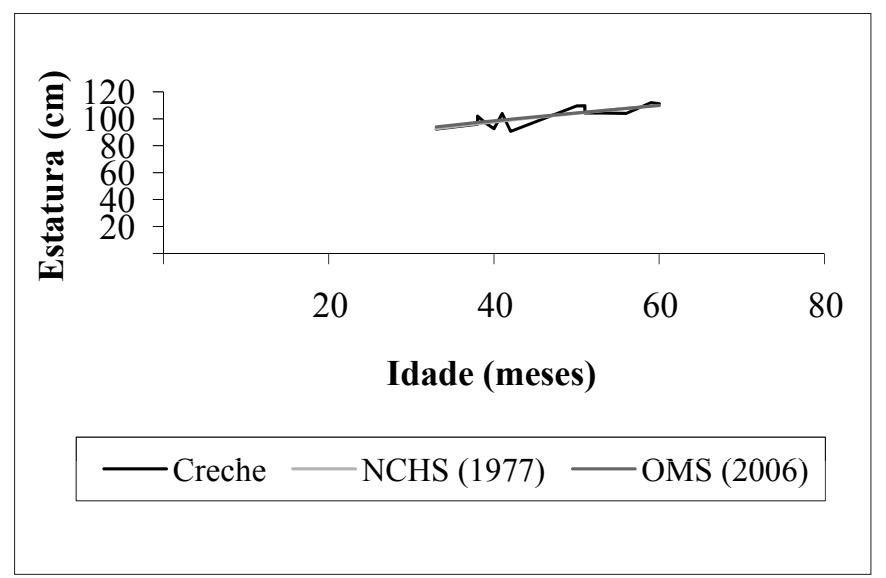

Gráfico 2 - Comparação da curva de estatura para idade observada nos meninos da creche com as curvas NCHS e OMS

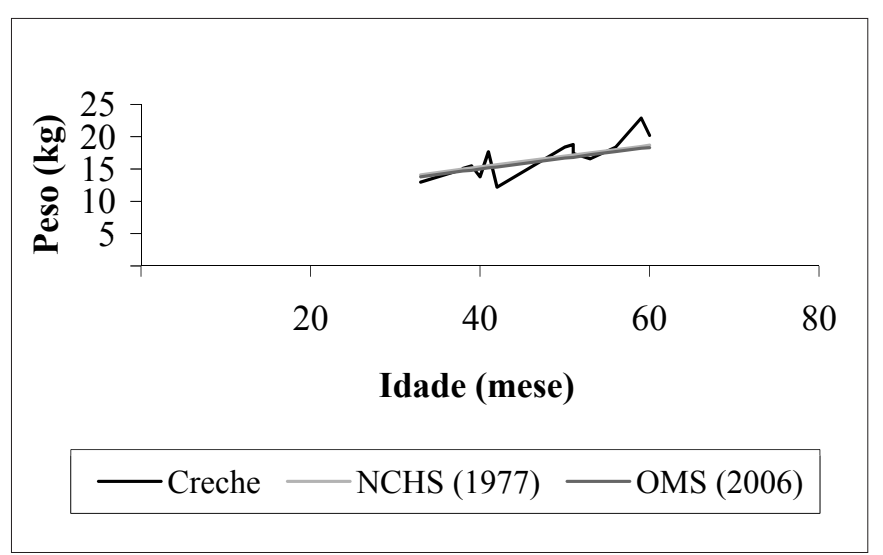

Gráfico 4 - Comparação da curva de peso para idade observada nos meninos da creche com as curvas NCHS e OMS

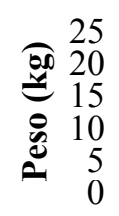

150

Estatura (cm)

- Creche

Gráfico 6 - Comparação da curva de peso e estatura observada nos meninos da creche com as curvas NCHS e OMS 
midade entre os valores do índice E/I para ambos os sexos, o que não ocorreu com as curvas dos índices P/I e P/E. Para estes índices, as meninas analisadas apresentaram maior média de peso do que os meninos.

Comparando-se os índices $\mathrm{P} / \mathrm{I}$ e $\mathrm{P} / \mathrm{E}$, observou-se maior sensibilidade do parâmetro $\mathrm{P} / \mathrm{I}$ na identificação dos préescolares com risco de baixo peso e uma maior sensibilidade do P/E na determinação das crianças obesas.

De acordo com o nível socioeconômico, constatou-se que, do total de crianças, 16 (49\%) pertenciam à classe econômica C, 15 (46\%) à classe D e duas (6\%) à classe E. A escolaridade do chefe da família de cada criança mostrou a seguinte distribuição: seis $(18,2 \%)$ analfabetos, oito $(24,2 \%)$ possuíam o primário completo, seis $(18,2 \%)$ com o ginasial completo e $13(39,4 \%)$ com o ensino médio completo.

\section{Discussão}

O fato de não se ter observado nenhum caso de desnutrição (escore $\mathrm{Z}_{<-2}$ ), de acordo com os indicadores P/I, P/E e E/I nas crianças da creche, pode ser atribuído a cinco aspectos básicos: expansão da melhoria de condições de saneamento básico; expansão do acesso às condições básicas de saúde; progressos moderados na renda familiar nacional; oferta de programas de suplementação alimentar e esforço da direção da creche em oferecer às crianças uma alimentação relativamente equilibrada, na qual estão incluídos todos os grupos de alimentos ${ }^{(14,15)}$. Cabe mencionar que o maior risco de deficit estatural e ponderal ocorre em torno do terceiro mês de vida e cai vagarosamente ao redor do terceiro ano de vida ${ }^{(16)}$. Isso também pode ter contribuído para o fato de não ter sido observado nenhum deficit (escore $Z_{<-2}$ ), de acordo com os indicadores utilizados, visto que a população da creche engloba pré-escolares entre três e seis anos de idade.

Sabe-se que o gênero é um fator biológico que interfere no estado nutricional, determinando o crescimento e o estoque energético diferenciado entre o sexo feminino e o masculino, ou seja, menor crescimento estatural e acúmulo energético na forma de gordura para o primeiro e maior crescimento estatural e maior aporte protéico para o segundo. Entretanto, pode-se observar semelhança nas variáveis peso e estatura quando as crianças foram comparadas segundo o gênero. Isso se deve ao fato de as crianças estudadas ainda não estarem na fase púbere (em torno dos 13 anos de idade), quando tais diferenças começam a se manifestar ${ }^{(17)}$.

Os resultados encontrados neste trabalho enquadram as crianças da creche no grupo de baixa prevalência de deficit estatural $(<20 \%)$ e ponderal $(<10 \%)$, segundo a proposta de classificação mundial para esta população ${ }^{(11)}$. Tais resultados se apresentaram, portanto, positivos na comparação com as referências mundiais para essa população no que se refere ao crescimento linear (E/I) e à massa corporal relativa à idade $(\mathrm{P} / \mathrm{I})$.

Ao comparar os dados deste estudo com a Pesquisa de Orçamentos Familiares, pode-se observar que, em relação ao indicador $\mathrm{P} / \mathrm{I}$, a ausência de baixo peso encontrada na creche diferiu dos resultados observados em crianças brasileiras $(4,6 \%)$ e dos estados da região Sudeste $(3,6 \%)$. Entretanto, é importante destacar que, diferentemente do presente estudo, na Pesquisa de Orçamentos Familiares foram incluídas crianças menores de três anos de idade, que constituem o grupo de maior risco para deficit ponderal ${ }^{(16,18)}$.

Em termos de sensibilidade dos índices, pode-se dizer que o índice P/I apresenta maior sensibilidade para identificar crianças menores de dez anos de idade com risco de desnutrição, visto que, nessa faixa etária, o peso é o parâmetro que tem maior velocidade de crescimento. $\mathrm{O}$ peso varia mais em função da idade do que do comprimento da criança, o que o torna mais sensível aos agravos nutricionais, sendo o primeiro a se modificar nestas circunstâncias ${ }^{(19)}$. Isto justifica a utilização desse índice pelo Ministério da Saúde a fim de identificar crianças com deficit ponderal.

$\mathrm{O}$ índice P/E mostra-se mais sensível para identificar crianças obesas maiores de três anos. Nessa fase, o crescimento global (peso e estatura) é mais lento e constante, predominando o estatural, fazendo com que o peso da criança varie mais em função de sua estatura do que da idade ${ }^{(19)}$. Desta forma, a avaliação da relação peso e estatura, com valores maiores de escore $\mathbf{Z}+2$, associa-se melhor com a adiposidade corporal $\mathrm{e}$, consequentemente, com a obesidade infantil.

Ao comparar os resultados de um estudo realizado pelo Departamento de Nutrição da Universidade de Brasília envolvendo crianças de três creches que fazem parte do projeto de extensão comunitária ${ }^{(20)}$ com a população da creche Maria José Santana, pôde-se observar o mesmo percentual de crianças obesas (6\%), segundo o indicador P/E. Como a OMS recomenda a utilização do índice P/E para obesidade e esta é mais freqüiente em maiores de três anos na maioria dos estudos ${ }^{(21,22)}$, a prevalência similar de obesidade nos dois estudos é esperada, principalmente em uma população que se caracteriza por famílias pertencentes às classes econômicas $C$, $\mathrm{D}$ e $\mathrm{E}$, com um pequeno grau de instrução. Destaca-se que a prevalência de sobrepeso (21\%) encontrada nas crianças avaliadas foi superior aos resultados de um outro estudo 
realizado com as crianças das creches municipais de Duque de Caxias (3\%), segundo o índice $\mathrm{P} / \mathrm{I}^{(23)}$.

Sendo assim, pode-se dizer que, as crianças da creche não apresentaram um resultado desejável nos quesitos sobrepeso e obesidade, tanto na comparação em nível mundial e nacional, quanto em nível regional e municipal. A alta prevalência de sobrepeso e obesidade observada pode se relacionar a dois aspectos: atividades em classe e atividades extra-classe, ambas influenciadas pelos fatores ambientais, como a ingestão energética excessiva e a atividade física diminuída ${ }^{(24)}$.

O percentual de pré-escolares com risco de baixa estatura (21\%) foi superior ao encontrado em outros dois estudos: um com crianças matriculadas em 31 creches municipais de Maringá, Paraná (10\%) e outro com crianças das creches municipais de Viçosa, Minas Gerais (15\%), podendo ser reflexo de piores condições ambientais e nutricionais ${ }^{(20,25)}$. Estes dados também podem explicar a prevalência de obesidade, já que as crianças não cresceram adequadamente em estatura nos primeiros anos de vida e o peso adquirido ficou em desequilíbrio para a pouca estatura.

Conforme dados do NCHS, existe uma margem esperada de incidência de desnutrição de até $2,3 \%$. Pôde-se observar que, na população da creche, tal margem não foi ultrapassada, apesar da elevada prevalência de risco de baixo peso e baixa estatura ${ }^{(12)}$. Por outro lado, a obesidade se mostrou como alteração nutricional preocupante, visto que o valor

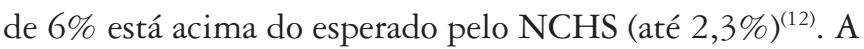
prevalência de sobrepeso também foi alarmante, uma vez que este dado é um forte indício do alto risco de maiores taxas de obesidade no futuro.

A comparação da prevalência de deficit de peso encontrado com os resultados da Pesquisa de Orçamentos Familiares mostrou que as crianças da creche analisada apresentaram resultado favorável, tanto na comparação em nível nacional quanto regional. Acredita-se que os determinantes socioeconômicos influenciaram o estado nutricional dos pré-escolares estudados, visto que as alterações nutricionais observadas estão relacionadas ao nível de instrução dos responsáveis e às condições econômicas das famílias. Conclui-se que os reflexos da transição nutricional foram notados neste estudo, no qual se encontrou ausência de deficits pôndero-estaturais indicativos de desnutrição e um considerável excesso de peso.

Cabe mencionar as várias limitações do estudo, destacandose as perdas de pacientes por questões éticas, as quais limitaram o número de crianças incluídas na análise e a ausência de informação sobre o consumo alimentar, que poderia complementar a análise da antropometria. Finalmente, por ser um estudo transversal, a relação de causalidade fica comprometida, mas, sem dúvida, gera hipóteses a serem testadas em outras pesquisas com delineamento adequado para estabelecer este tipo de relação. Destaca-se, a contribuição da presente investigação para exemplificação do perfil de alterações nutricionais que têm ocorrido na população infantil brasileira.

\section{Agradecimentos}

Ao diretor da creche Maria José Santana, Reverendo José de Santana Santos, que confiou na seriedade do trabalho, dando livre acesso aos compartimentos internos da Instituição para a realização da pesquisa. À coordenadora da creche Maria José Santana, Elizete Olímpio da Silva, que me apoiou em todas as atividades desenvolvidas com as crianças, sempre com um belo sorriso no rosto, sendo solícita em todos os meus pedidos.

\section{Referências bibliográficas}

1. Brasil - Ministério do Planejamento, Orçamento e Gestão. Instituto Brasileiro de Geografia e Estatística (IBGE). Estudo nacional de despesa familiar. Brasília: IBGE, 1974.

2. Brasil - Ministério da Saúde. Instituto Nacional de Alimentação e Nutrição (INAN). Pesquisa nacional sobre saúde e nutrição: perfil de crescimento da população brasileira de 0 a 25 anos. Brasília: INAN, 1990.

3. Brasil - Ministério do Planejamento, Orçamento e Gestão. Instituto Brasileiro de Geografia e Estatística (IBGE). Pesquisa de orçamentos familiares 20022003: antropometria e análise do estado nutricional de crianças e adolescentes no Brasil. Brasília: IBGE, 2006.

4. Kac G, Velásquez-Meléndez G. A transição nutricional e a epidemiologia da obesidade na América Latina. Cad Saude Publica 2003;19 (Suppl 1):S4-5.
5. Oliveira RC. A transição nutricional no contexto da transição demográfica e epidemiológica. Rev Min Saude Pub 2004;5:16-23.

6. Biscegli TS, Corrêa CE, Romera J, Hernandez JL. Avaliação do estado nutricional e prevalência de carência de ferro em crianças freqüentadoras de uma creche. Rev Paul Pediatr 2006;24:323-9.

7. National Center for Health Statistics. Growth curves for children birth-18 years of age, United States. Vital and health statistic: series 11, n 165. Washington, DC: US Government Printing Office, 1977.

8. OMS - Organização Mundial de Saúde [homepage on the internet]. La OMS difunde un nuevo patrón de crecimiento infantil [cited 2006 Nov 24]. Available from: http://www.who.int/mediacentre/news/releases/2006/pr21/ es/index.html 
9. ABEP - Associação Brasileira de Empresas de Pesquisa [homepage on the Internet]. Critério de classificação econômica Brasil [cited 2006 Oct 18]. Available from: http://www.abep.org

10. Brasil - Ministério da Indústria, do Comércio e do Turismo. Instituto Nacional de Metrologia, Normalização e Qualidade Industrial - INMETRO. Portaria $n^{\circ}$ 236, 1994.

11. Lohman TG, Roche AF, Martorell R. Anthropometric standardization reference manual. Champaign: Human Kinetics Books; 1988.

12. Shils ME, Olson JA, Shike M, Ross AC. Tratado de nutrição moderna na saúde e na doença. $9^{a}$ ed. São Paulo: Manole; 2003.

13. World Health Organization. Physical status: the use and interpretation of anthropometry. Report of a WHO Expert Committee. Technical report series, 854. Geneva: WHO, 1995.

14. Monteiro CA. Velhos e novos males da saúde no Brasil: evolução do país e suas doenças. São Paulo: Hucitec; 1995.

15. Escobar MS. Diagnóstico nutricional de crianças menores de cinco anos do Município de Casimiro de Abreu [monografia de especialização em Nutrição Materno Infantil]. Rio de Janeiro: UERJ; 2002.

16. Freitas SN. Fatores determinantes do estado nutricional de crianças menores de 60 meses no município de Ouro Preto-Minas Gerais [tese de mestrado]. Rio de Janeiro: UFRJ; 1998.

17. Saúde na internet [homepage on the Internet]. Alimentação na fase préescolar. [cited 2006 Nov 11]. Available from: http://www.saudenainternet.com. br/portal_saude/alimentacao-na-fase-pre-escolar.php
18. Sigulem DM, Devincenzi MV, Lessa AC. Diagnóstico do estado nutricional da criança e do adolescente. J Pediatr 2000;76 (Suppl 3):S275-84.

19. Tuma RCFB, Costa THM, Schmitz BAS. Avaliação antropométrica e dietética de pré-escolares em três creches de Brasília, Distrito Federal. Rev Bras Saúde Mater Infant 2005;5:419-28.

20. Kuranishi LT, Silva FM, Machado CF, Cestari Junior LA, Sekiyama JY, Mazuti RMR et al. Avaliação do estado nutricional de pré-escolares matriculados nas creches municipais de Maringá-PR no ano de 2001 [monograph on the Internet]. Paraná: UEM; 2001. [cited 2008 Jul 19]. Available from: http://www. saudebrasilnet.com.br/premios/saude/premio2/trabalhos/025.pdf

21. Fisberg RM, Marchioni DML, Cardoso MRA. Estado nutricional e fatores associados ao déficit de crescimento de crianças freqüentadoras de creches públicas do Município de São Paulo, Brasil. Cad Saude Publica 2004;20: 812-7.

22. Guimarães LV, Barros MBA. As diferenças de estado nutricional em pré-escolares de rede pública e a transição nutricional. J Pediatria 2001;77:381-6.

23. Junior ESM. Projeto de implantação da vigilância nutricional nas creches municipais de Duque de Caxias [monografia]. Duque de Caxias: SMSDC; 2004.

24. Silva GAP, Balaban G, Motta MEFA. Prevalência de sobrepeso e obesidade em crianças e adolescentes de diferentes condições socioeconômicas. Rev Bras Saude Mater Infant 2005;5:53-9.

25. Castro TG, Novaes JF, Silva MR, Costa NMB, Franceschini SCC, Tinôco ALA et al. Caracterização do consumo alimentar, ambiente socioeconômico e estado nutricional de pré-escolares de creches municipais. Rev Nutr 2005;18:321-30. 